Missed Diagnosis

\title{
Pulmonary endometriosis mimicking an acute abdomen
}

\author{
R.A. Grunewald and J. Wiggins
}

Thoracic Medicine, Brompton Hospital, Fulham Road, London SW3 6HP, UK.

\begin{abstract}
Summary: A case of pulmonary endometriosis is reported, to illustrate the classic catamenial symptoms of the disease. The patient also developed symptoms and signs suggesting a perforated abdominal viscus; laparotomy was normal and the features may have resulted from a previously unreported effect of diaphragmatic endometrial foci.
\end{abstract}

\section{Introduction}

Intrathoracic endometriosis is a rare condition of premenopausal women which can present with either asymptomatic pulmonary nodules or with catamenial (occurring with menstruation) symptoms, such as haemoptysis, haemothorax and pneumothorax. We report an unusual case in which a patient with thoracic endometriosis developed clinical and radiological signs suggesting a perforated abdominal viscus, although at laparotomy all abdominal and pelvic organs were normal.

\section{Case report}

During a menstrual period, a 28 year old woman developed right-sided chest and shoulder tip pain followed by sudden onset of epigastric pain with vomiting. During the preceding 18 months she had suffered recurrent pneumothoraces, haemoptysis, chest pain and fever associated with the onset of her menstrual periods. She underwent bilateral apical pleurectomies after 6 months, but continued to have catamenial chest pain with pyrexia, haemoptysis and haemothorax and a firm diagnosis was not made, although a diagnosis was volunteered by the patient's fiancé, himself a surgeon.

On examination, she was pyrexial, semi-conscious and shocked (blood pressure $80 / 0 \mathrm{~mm} \mathrm{Hg}$ ), with a tachycardia (pulse $125 / \mathrm{min}$ ) but no cyanosis. The abdomen was diffusely tender, maximally in the epigastrium and right upper quadrant, and there was rebound tenderness and guarding. Bowel

Correspondence: J. Wiggins, M.D., M.R.C.P.

Accepted: 6 July 1988 sounds were reduced. The full blood count revealed a white cell count of $10.6 \times 10^{9} / 1$, and a normal haemoglobin concentration. Serum amylase and electrocardiogram were normal. The chest radiograph showed free gas under the right hemi-

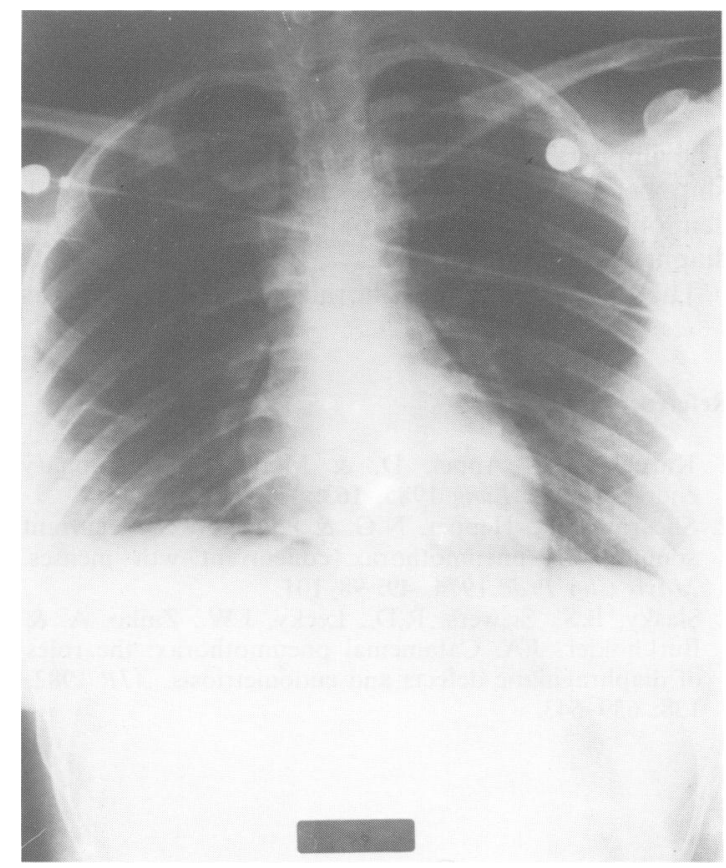

Figure 1 Pre-operative chest radiograph showing gas under the right hemidiaphragm.

(C) The Fellowship of Postgraduate Medicine, 1988 
diaphragm with blunting of the right costophrenic angle (which was present on previous and subsequent films) but no evidence of pneumothorax (Figure 1). A clinical diagnosis of perforated viscus was made. At laparotomy turbid fluid was found in the right paracolic gutter. All abdominal viscera including uterus and adnexae were normal and there was no evidence of previous peptic ulcer disease, though a few adhesions were found around the caecum. There was no evidence of abdominal or pelvic endometriosis, although the diaphragm was not specifically examined. Cultures of blood and peritoneal fluid were not performed.

She made an uneventful postoperative recovery on no specific therapy. However, 10 days after her laparotomy she began to menstruate and had a further episode of haemoptysis, pyrexia, chest pain and right sided haemothorax. Right-sided open lung biopsy was performed which revealed a cavity at the right base lined by thickened pleura containing fresh haemorrhage and eosinophilic infiltrate consistent with endometriosis, though no endometrial tissue was identified. She was subsequently commenced on treatment with buserelin, a luteinizing hormone releasing hormone analogue, to suppress menstruation. Four months after starting treatment she has had no further recurrence of her symptoms, which before treatment had occurred with every menstrual period for 18 months.

\section{Discussion}

The important feature of this case is the relationship between intrathoracic endometriosis and sudden onset of abdominal pain with gas under the diaphragm.

The diagnosis of intrathoracic endometriosis was

\section{References}

1. Karpel, J.P.., Appel, D. \& Merav, A. Pulmonary endometriosis. Lung 1985, 163: 151-159.

2. Shearin, R.P., Hepper, N.G. \& Payne, W.S. Recurrent spontaneous pneumothorax concurrent wth menses. Mayo Clin Proc 1974, 49: 98-101.

3. Slasky, B.S., Siewers, R.D., Lecky, J.W., Zajko, A. \& Burkholder, J.A. Catamenial pneumothorax: the roles of diaphragmatic defects and endometriosis. AJR 1982, 138: 639-643. based on the characteristic clinical features of the $\frac{3}{8}$ disease: multiple catamenial pneumothoraces, hae- $\bigcirc$ mothoraces and haemoptyses associated with pyr.exia and dyspnoea. ${ }^{1}$ Diagnostic biopsy material $\vec{\equiv}$ may be hard to obtain especially immediately after $-\overrightarrow{0}$ menstruation because foci of endometriosis are $\frac{}{5}$ small and evanescent, ${ }^{2}$ although in this case the $\overline{\bar{m}}$ histology was entirely consistent with the diagnosis. There are two possible mechanisms whereby tho- $\frac{0}{0}$ racic endometriosis may have produced peritonism is and sub-diaphragmatic air. Diaphragmatic fenest- $\overrightarrow{0}$ rations are a characteristic feature of thoracic endometriosis $^{3}$ and may result from perforation of the $\vec{\omega}$ diaphragm by endometrial foci, though they occur $\frac{}{0}$ occasionally in normal individuals. Such a fenestration may have allowed a catamenial haemopneumothorax to drain into the abdomen and + produce sudden onset of peritonism. Alternatively, of a pulmonary endometrial focus may have eroded. adherent pleura and diaphragm to produce an abdomino-pulmonary fistula. Pneumothorax was prevented by the previous bilateral apical pleurectomies.

Such a case presentation poses a difficult management problem for the surgeon, because even $\Phi$ when the diagnosis of endometriosis is very probable ${ }^{4}$ a coincidental perforated viscera has to be $\mathbb{C}$ excluded. Thoracic endometriosis can occur in the absence of abdominal or pelvic disease; ${ }^{5}$ this shour not prevent early diagnosis of a condition for whi effective therapy may be available.

\section{Acknowledgement}

We thank Professor M. Turner-Warwick for permission to $\overline{\bar{o}}$ report the case, and Miss T. Chudleigh for secretarial assistance.
4. Crutcher, R.R., Waltuch, T.L. \& Blue, M.E. Recurring spontaneous pneumothorax associated with menstrua- 8 tion. J Thorac Cardiovasc Surg, 1967, 54: 599-602.

5. Barrocas, A. Catamenial pneumothorax: case report and a review of the literature Am Surg 1979, 45: 340-343. 